\title{
Gene silencing below the immune radar
}

\author{
Gunther Hartmann
}

Institute of Clinical Chemistry and Pharmacology, University of Bonn, Bonn, Germany.

\begin{abstract}
In vertebrates, the detection of viral nucleic acids is the first step toward innate and subsequent adaptive antiviral immune responses. A sophisticated, protein receptor-based sensor system has evolved to recognize viral nucleic acids and to trigger a variety of antiviral defense mechanisms. The more we learn about this elaborate sensor system, the more it becomes evident how difficult it is to introduce exogenous nucleic acids such as siRNA into cells without triggering antiviral immunoreceptors. In this issue of the JCI, Judge and colleagues provide evidence that siRNA can be designed and delivered in a way that allows specific and successful silencing of target genes in tumor cells in vivo, leading to tumor cell death and prolonged survival of tumor-bearing mice in the absence of immune activation (see the related article beginning on page 661). This study represents a major technological advance, setting new standards for well-controlled siRNA applications in vivo, and has the potential to guide clinical development toward siRNA therapeutics with well-defined and selective gene-silencing activities.
\end{abstract}

Nature has evolved two major antiviral defense strategies: one is based on degradation of long, viral dsRNA molecules to short, viral dsRNA molecules and amplification thereof, resulting in RNAi-mediated cleavage of complementary viral RNA sequences inside the cell; the other antiviral defense strategy requires the detection of viral nucleic acid by specialized protein receptors, the activation of which leads to innate and adaptive antiviral immune responses. While the first antiviral strategy makes use of Watson-Crick base pairing and enzymatic cleavage of complementary viral sequences, the second uses proteinto-nucleic acid binding, initiating signal transduction pathways, resulting in the activation of a number of downstream antiviral mechanisms. Although both principles are fundamentally different, they have the very same aim, that is, to destroy viral genetic material and prevent spreading of viral infection. It is important to note that, in addition to antiviral defense, the biological principle of RNAi plays a key role in gene regulation (e.g., microRNA); furthermore, the antiviral defense in higher vertebrates predominantly relies not on RNAi but on receptor-mediated recognition of viral nucleic acids. It almost seems that the

Conflict of interest: The author has declared that no conflict of interest exists.

Nonstandard abbreviations used: RIG-I, retinoic acid-inducible gene I; SNALP, stable nucleic acid lipid particle(s).

Citation for this article: J. Clin. Invest. 119:438-441 (2009). doi:10.1172/JCI38475. use of RNAi as an antiviral strategy and the use of receptor-mediated recognition are mutually exclusive. RNAi machinery in the context of gene regulation provides the basis for using exogenous siRNAs to silence specific genes. Physiologically, siRNAs are generated from microRNA precursors generated in the nucleus (1) (Figure 1). The receptors specializing in detecting viral nucleic acids obviously ignore these natural, short RNA molecules originating from the nucleus. However, the therapeutic use of synthetic siRNA requires delivery of exogenous siRNA across the cell membrane to the cytosol of the target cell. In an organism such as the worm, which relies on RNAi and not on receptor-mediated recognition for antiviral defense, delivery of exogenous siRNA does not alert the cell to the presence of a virus. However, in higher vertebrates with receptor-mediated recognition of viral RNA in place, entrance of exogenous RNA is detected and turns on an alarm (2) (Figure 2). Consequently, in order for RNAi technology to be successfully applied in higher vertebrates, we must first design and then determine how to deliver siRNA that allows for the activation of the RNAi machinery without alerting the target cell of an apparent viral infection (3).

\section{Detection of SiRNA by TLR7 and RIG-I}

Before the era of siRNA, long dsRNA was be applied to lower organisms with no
In higher vertebrates, the presence of the used to mediate RNAi, but this could only protein receptor-mediated mechanism for the recognition of viral RNA, for example, worms such as Caenorbabditis elegans (4). An important advance in the RNAi field came from the lab of Thomas Tuschl, who made the observation that short, dsRNA oligonucleotides seemed to be ignored by one of the dsRNA-detecting receptors, PKR, even in higher vertebrates, while maintaining the ability to trigger $\operatorname{RNAi}(5,6)$. This pioneering work generated great enthusiasm about the use of siRNA for functional genomics and for therapeutic application. Then, in 2005, it became clear that the vertebrate immune system possessed a receptor for the detection of such short dsRNA molecules, TLR7 (7) (Figure 2). Detection of siRNA by TLR7 is sequence dependent, but the majority of randomly selected siRNAs activate TLR7 to some degree. Besides siRNA, TLR7 detects long and short single-stranded RNA (ssRNA) with a preference for G- and U-rich sequences (7-9). The reason that Tuschl's group did not detect immune activation by siRNA in their experiments is that the expression of TLR7 (first described in ref. 10) is largely restricted to B cells and to a highly specialized immune cell subset, the plasmacytoid DCs (11). Consequently, TLR7 is not expressed in most cell lines in which siRNA is tested. The absence of TLR7 in most cell types allows the undertaking of specific gene-silencing experiments in these cells without eliciting TLR7-mediated antiviral responses. However, as soon as an siRNA found to be target specific in vitro is administered in vivo, TLR7expressing cells are generated and TLR7mediated antiviral responses obscure the specificity of silencing.

Soon it became clear that introducing 2'O-methyl modifications of RNA reduced activation via TLR7 $(3,12)$. However, introducing 2'O-methyl modifications to the antisense strand also reduced silencing activity, and without modification of the antisense strand it seems impossible to completely eliminate TLR7 ligand activity. Moreover, irrespective of 2'O-methyl modifications, TLR3 ligand activity and suppression of angiogenesis was reported as a class effect of siRNA (13). Furthermore, 


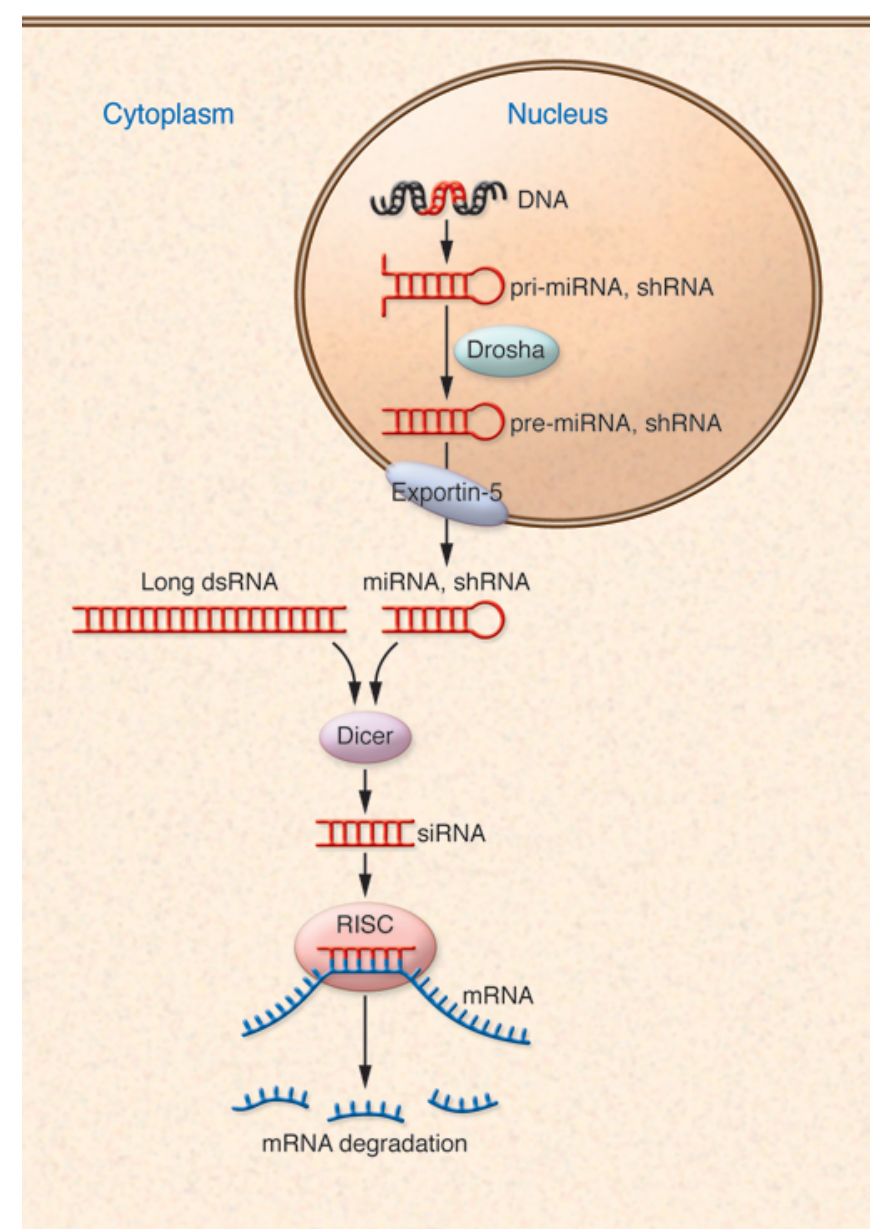

one group claimed that the cytosolic helicase retinoic acid-inducible gene I (RIG-I) was able to detect short dsRNA oligonucleotides such as siRNA independently of a 5' triphosphate group (14). Others reported that $5^{\prime}$ triphosphate was required in order for short RNA molecules to activate RIG-I $(12,15-17)$. As a consequence of these findings, the initial enthusiasm about the therapeutic utility of siRNA turned to skepticism as researchers wondered whether target-specific siRNA-mediated silencing was achievable at all without simultaneous activation of RNA immunoreceptors.

\section{Modification of siRNA to escape immunorecognition}

In this issue of the JCI, Judge et al. report a major advance in the design and application of siRNA (18) (Figure 2). They demonstrate that siRNA can be designed and delivered to a target organ so that it is ignored by nucleic acid-detecting receptors, even by sensitive measures, and that such siRNA specifically and efficiently silences target RNA expression and sup- presses the biological function of the target protein in vivo. The experimental design of this study sets new standards for siRNAmediated, target-specific gene silencing in vivo. The starting point of their study is the observation that 2'O-methyl modification of the sense strand is sufficient to avoid the induction of systemic cytokines such as type I IFN but not sufficient to eliminate the induction of IFN-induced genes (IFN-induced protein with tetratricopeptide repeats 1 [IFIT1] RNA expression was utilized as a sensitive measure) in liver tissue of mice treated with siRNA. One of the key findings in the study is that it is possible to select positions in the antisense strand for 2'O-methyl modification, which abrogates IFN induction, while silencing is fully maintained. In such siRNA molecules, 6 to 8 bases at the $5^{\prime}$ end of the antisense strand remained without modification. The lack of immunostimulatory activity was confirmed by the lack of IL- 6 and IFN- $\alpha$ induction in DCs generated from murine bone marrow in the presence of FMS-like tyrosine kinase 3 (Flt3)

\section{Figure 1}

Antiviral and gene regulatory function of RNAi. Long, viral dsRNA in the cytoplasm is cleaved to short, viral dsRNA (siRNA) by Dicer. MicroRNA (miRNA), a class of noncoding RNA molecules involved in gene regulation, is generated by Drosha-mediated cleavage from pri-miRNA transcribed in the nucleus, exported by Exportin- 5 to the cytoplasm, and then cleaved by Dicer, resulting in siRNA. Similarly to physiological pri-miRNA, shRNA introduced to cells via gene transfer enters the same pathway (Drosha, Dicer), resulting in the release of the corresponding siRNA. siRNA produced by both pathways binds to RNA-induced silencing complex (RISC), and the sense strand is released from the RISC complex. Complementary binding of the antisense strand to target sequences guides RISC to cleave the target sequence (resulting in mRNA degradation) or, in the absence of full complementarity to the target sequence, inhibits its translation. ligand, the lack of systemic IFN- $\alpha$ in the serum, and the lack of IFIT1 RNA induction in liver and spleen.

An elegant experimental setting was chosen to confirm in vivo silencing of such siRNA (18). The two selected cell-cycle proteins, polo-like kinase 1 (PLK1) and kinesin spindle protein (KSP), showed almost no baseline expression in normal liver but high expression in tumors. Furthermore, functional inhibition led to characteristic morphological changes. Thus, in mice carrying liver tumors, silencing of the target genes as measured in total target tissue (liver) reflects silencing in tumor cells, and this was confirmed by the detection of characteristic morphological changes in tumor cells. The tumor models included a xenograft model (Hep3B) in immunodeficient mice (SCID/beige mice) and a syngeneic model (Neuro2a) in immunocompetent A/J mice. These two models nicely complement each other: immune-independent silencing through siRNA was tested in immunodeficient mice; specificity of silencing and the lack of immune activation was confirmed 


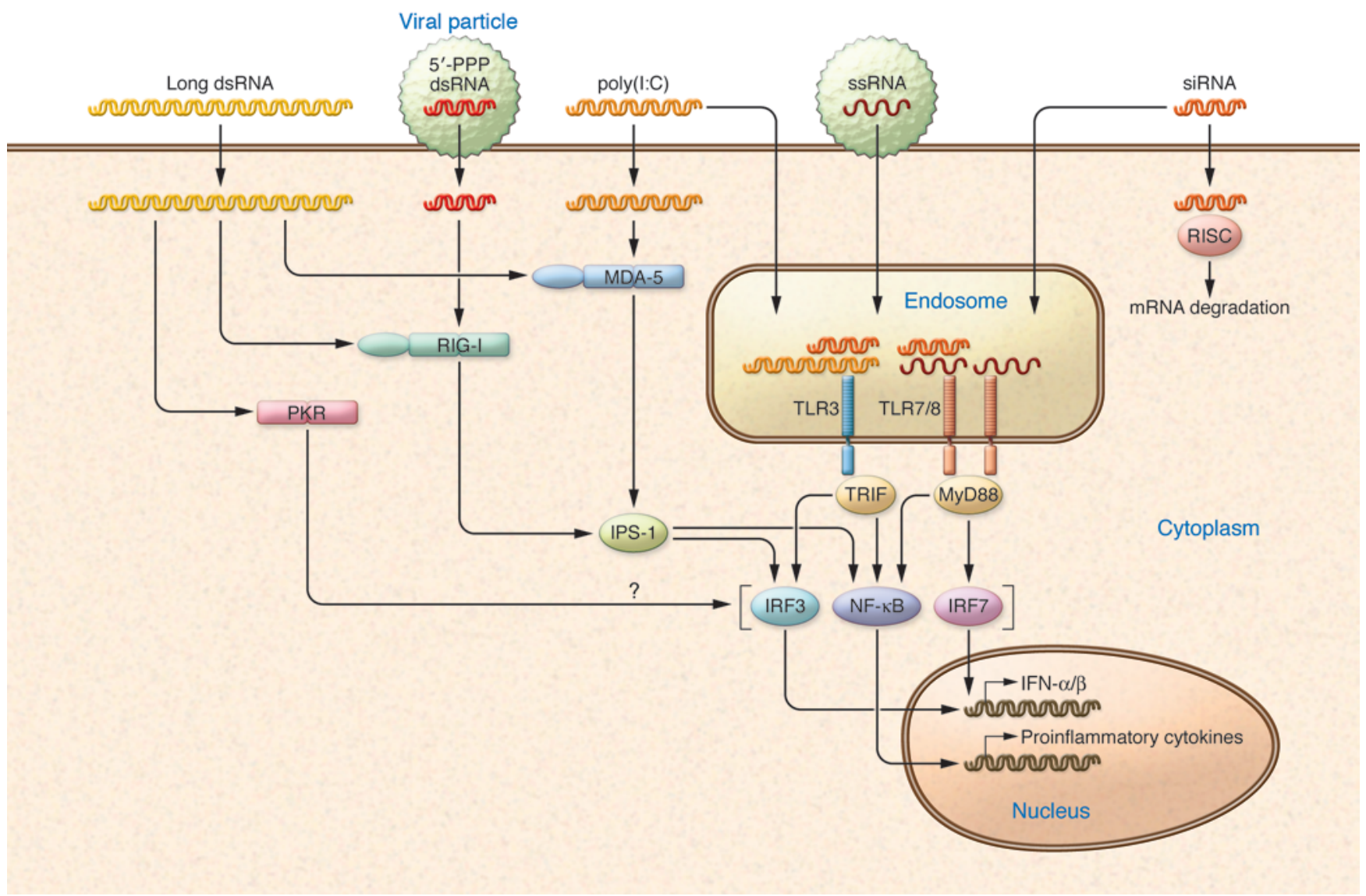

\section{Figure 2}

Immunorecognition of RNA. RNA delivered to the endosome is detected by TLR3, in the case of long dsRNA or its mimic poly(I:C) and short dsRNA, or by TLR7, in the case of siRNA and single-stranded RNA (ssRNA). RNA delivered to the cytosol can be recognized by cytosolic helicases. The RNA helicase RIG-I detects RNA carrying a triphosphate group at the $5^{\prime}$ end (5'-PPP) and possibly blunt-end short RNA and intermediate dsRNA. Melanoma differentiation-associated protein-5 (MDA-5) detects long dsRNA. Long dsRNA also binds to RNA-binding PKR, but activation of IRF3 and IRF7 and subsequent type I IFN induction by PKR is controversial (as indicated by question mark). TLRs signal via TIR domain-containing adapter inducing IFN- $\beta$ (TRIF) (in the case of TLR3) to activate IRF3 and NF- $\mathrm{kB}$ or via MyD88 (in the case of TLR7) to activate IRF7 and NF-кB. RIG-I and MDA-5 signal via IFN- $\beta$ promoter stimulator-1 (IPS-1) to induce IRF7 and NF- $\mathrm{kB}$. Depending on the cell type and its receptor expression pattern (e.g., ECs, myeloid DCs), recognition of RNA leads to the production of type I IFNs (e.g., IFN- $\alpha / \beta)$, and of proinflammatory cytokines or may lead to the modulation of cell-specific functions. In the study reported by Judge et al. (18) in this issue of the $\mathrm{JCl}$, immunorecognition of siRNA is avoided by chemical modification and by the specific mode of delivery.

in mice with an intact immune system. Together, the selected targets and tumor models allowed the authors to pinpoint functional silencing in tumor cells in vivo.

\section{Successful delivery of siRNA}

There is broad consensus that delivery is key to successful siRNA application in vivo. In their study, Judge et al. (18) provide evidence for successful use of stable nucleic acid lipid particles (SNALP) for delivery of siRNA to the liver. Furthermore, they demonstrate that silencing and antitumor activity in subcutaneous tumors can be improved by modifying the lipid composition of SNALP (i.e., incorporating PEG lipids with longer alkyl chains). Effective silencing in the sub- cutaneous location suggests that modified SNALP formulations may have the potential to facilitate silencing in other tissues and locations. Nevertheless, with this study, formal proof is currently provided only for the liver and for the subcutaneous location, but at least for these two locations, the delivery issue seems to be sufficiently resolved.

But what about the TLR3-dependent antiangiogenic class effect of siRNA (13)? TLR3 is expressed in a variety of cell types including myeloid DCs and ECs. TLR3 signals via TIR domain-containing adapter inducing IFN- $\beta$ (TRIF), leading to the induction of IFN- $\beta$ in immune cells. In the current study by Judge and colleagues (18), the absence of a detectable IFN response in healthy liver, despite effective silencing in liver tumors, supports a TLR-independent effect. However, it would be interesting to know whether the siRNA in conjunction with SNALP delivery provokes a TLR3dependent antiangiogenic effect similar to the effect of naked siRNA reported by Kleinman and colleagues (13). In fact, in the Kleinman and colleagues' work, siRNA induced TLR3-dependent suppression of angiogenesis in the absence of a type I IFN response. One could speculate that ECs respond to low-level TLR3 activation by reducing angiogenesis even before they start making type I IFN. Myeloid DCs are expected to produce IFN- $\beta$ upon TLR3 activation, but in myeloid DCs, TLR3 is expressed in 
a different compartment (endosomal compared with surface in ECs), and the level of stimulation by short dsRNAs such as siRNA may not be sufficient to induce considerable levels of IFN- $\beta$ in these cells. If this is the case, then the antiangiogenic effect of TLR3 may indeed contribute to the in vivo antitumor effect of the siRNA seen in the current study by Judge et al. (18). The extent of such contributions may be low; however, a possible antitumor effect of the control siRNA carrying the same type of modifications cannot be deduced from the data presented, since the control without siRNA is not depicted in most of the figures related to tumor therapy in vivo.

Unlike TLR3, the helicase RIG-I is located in the same compartment as the enzyme complex mediating siRNA-induced silencing (RNA-induced silencing complex [RISC]). The siRNA used by Judge et al. (18) had $5^{\prime}$ overhangs, which does not support activation of RIG-I (14). Indeed, there is no hint in this study that RIG-I activation participates in the antitumor effect. Furthermore, it is interesting to note that no reduction in white blood cell counts or liver toxicity was observed. This is an important observation, considering that SNALP transfer nucleic acid cargo over intact cell membranes. Disturbance or leakage of cellular or endosomal membranes activates the inflammasome (19-21). However, the analysis of IFN- $\alpha$ and IL- 6 as used in the Judge et al. study is not adequate to detect inflammasome activation. It would be interesting to know whether IL-1 $\beta$ and IL-18 are induced by SNALP-delivered siRNA. Since inflammasome-dependent, caspase-1-mediated release of IL- $1 \beta$ and IL-18 requires the presence of the proforms of IL-1 $\beta$ and IL-18, such SNALP-dependent inflammasome activation may only be present in tumorbearing mice and may not be detectable in the healthy animals in which immunological activity of siRNA was assessed by Judge and colleagues. Even in the absence of IFN, inflammasome activation may contribute to the antitumor activity of siRNA. Along this line, it would be important to know whether the absence of a detectable IFN response in the healthy liver, as demonstrated in the current study, holds true for tumor-bearing livers. Livers carrying tumor tissue may show higher infiltration with preactivated immune cells and thus may be more susceptible to siRNA-induced immunostimulation. Although target-specific siRNA silences its target in tumor cells by site-specific cleavage (confirmed by rapid amplification of cDNA ends [RACE] technology) in vivo, quantification of the extent to which silencing of the target indeed contributes to in vivo therapeutic activity would require more controls than provided. Furthermore, specificity of silencing one versus the other cell-cycle protein target would be best controlled by simultaneous quantification of both corresponding target RNAs.

Comparison of tumors derived from tumor cells expressing the wild-type versus a mutated target sequence (mutation resulting in the same amino acid sequence but loss of target sequence) allows for the assessment of the relative contribution of silencing versus immunostimulation of an siRNA molecule (22). Several groups have shown that immune activation by RNA has antitumor activity, and thus it would be interesting to know how an immunostimulatory RNA oligonucleotide performs compared with a target-specific siRNA in the specific tumor models used in the Judge et al. study (18).

Nevertheless, based on the current study (18) and other recent work, the RNAi field should regain confidence that appropriate siRNA delivery and target-specific silencing of genes can be achieved in vivo. Some uncertainty remains as to whether non-RNAi-related effects such as antiangiogenesis or inflammasome activation or a type I IFN response in diseased tissues may add to the RNAi-based therapeutic activity of siRNA. With this methodological advance and the reported proof of principle for siRNA-mediated gene silencing in vivo, without doubt, the field will revive its enthusiasm for RNA-based therapeutics. With this development, the use of target-specific siRNA-mediated gene silencing in vivo in combination with the effects of other welldefined biological properties of RNA (such as TLR7- or RIG-I activation) is even more attractive, specifically for tumor therapy.

\section{Acknowledgments}

This study was supported by German Federal Ministry of Education and Research (Bundesministerium für Bildung und Forschung [BMBF]) BioFuture grant 0311896 and by Deutsche Forschungsgemeinschaft (DFG) grants SFB 670, SFB 704, KFO115, and KFO 177, awarded to G. Hartmann.

Address correspondence to: Gunther Hartmann, Institut für Klinische Chemie und Pharmakologie, Universitätsklinikum Bonn, Sigmund-Freud-Str. 25, D-53127 Bonn, Germany. Phone: 49-228-287-16080; Fax: 49-228-287-16094; E-mail: gunther. hartmann@ukb.uni-bonn.de.
1. Landgraf, P., et al. 2007. A mammalian microRNA expression atlas based on small RNA library sequencing. Cell. 129:1401-1414.

2. Schlee, M., Barchet, W., Hornung, V., and Hartmann, G. 2007. Beyond double-stranded RNA-type I IFN induction by 3 pRNA and other viral nucleic acids. Curr. Top. Microbiol. Immunol. 316:207-230.

3. Judge, A., and MacLachlan, I. 2008. Overcoming the innate immune response to small interfering RNA. Hum. Gene Ther. 19:111-124.

4. Fire, A., et al. 1998. Potent and specific genetic interference by double-stranded RNA in Caenorhabditis elegans. Nature. 391:806-811.

5. Elbashir, S.M., et al. 2001. Duplexes of 21-nucleotide RNAs mediate RNA interference in cultured mammalian cells. Nature. 411:494-498.

6. Elbashir, S.M., Martinez, J., Patkaniowska, A., Lendeckel, W., and Tuschl, T. 2001. Functional anatomy of siRNAs for mediating efficient RNAi in Drosophila melanogaster embryo lysate. EMBO J. 20:6877-6888.

7. Hornung, V., et al. 2005. Sequence-specific potent induction of IFN-alpha by short interfering RNA in plasmacytoid dendritic cells through TLR7. Nat. Med. 11:263-270.

8. Heil, F., et al. 2004. Species-specific recognition of single-stranded RNA via toll-like receptor 7 and 8 . Science. 303:1526-1529.

9. Diebold, S.S., et al. 2006. Nucleic acid agonists for Toll-like receptor 7 are defined by the presence of uridine ribonucleotides. Eur. J. Immunol. 36:3256-3267.

10. Du, X., Poltorak, A., Wei, Y., and Beutler, B. 2000. Three novel mammalian toll-like receptors: gene structure, expression, and evolution. Eur. Cytokine Netw. 11:362-371.

11. Hornung, V., et al. 2002. Quantitative expression of toll-like receptor 1-10 mRNA in cellular subsets of human peripheral blood mononuclear cells and sensitivity to CPG oligodeoxynucleotides. J. Immunol. 168:4531-4537.

12. Hornung, V., et al. 2006. $5^{\prime}$-Triphosphate RNA is the ligand for RIG-I. Science. 314:994-997.

13. Kleinman, M.E., et al. 2008. Sequence- and targetindependent angiogenesis suppression by siRNA via TLR3. Nature. 452:591-597.

14. Marques, J.T., et al. 2006. A structural basis for discriminating between self and nonself doublestranded RNAs in mammalian cells. Nat. Biotechnol. 24:559-565.

15. Cui, S., et al. 2008. The C-terminal regulatory domain is the RNA 5'-triphosphate sensor of RIG-I. Mol. Cell. 29:169-179.

16. Pichlmair, A., et al. 2006. RIG-I-mediated antiviral responses to single-stranded RNA bearing 5 '-phosphates. Science. 314:997-1001.

17. Saito, T., Owen, D.M., Jiang, F., Marcotrigiano, J., and Gale, M. 2008. Innate immunity induced by composition-dependent RIG-I recognition of hepatitis C virus RNA. Nature. 454:523-527.

18. Judge, A.D., et al. 2009. Confirming the RNAimediated mechanism of action of siRNAbased cancer therapeutics in mice. J. Clin. Invest. 119:661-673.

19. Hornung, V., et al. 2008. Silica crystals and aluminum salts activate the NALP3 inflammasome through phagosomal destabilization. Nat. Immunol. 9:847-856.

20. Muruve, D.A., et al. 2008. The inflammasome recognizes cytosolic microbial and host DNA and triggers an innate immune response. Nature. 452:103-107.

21. Petrilli, V., Dostert, C., Muruve, D.A., and Tschopp, J. 2007. The inflammasome: a danger sensing complex triggering innate immunity. Curr. Opin. Immunol. 19:615-622.

22. Poeck, H., et al. 2008. 5'-Triphosphate-siRNA: turning gene silencing and Rig-I activation against melanoma. Nat. Med. 14:1256-1263. 


\title{
Protein degradation in Parkinson disease revisited: it's complex
}

\author{
Han Li' and Ming Guo ${ }^{1,2}$ \\ 1UCLA ACCESS Graduate Program and 2Department of Neurology and Department of Molecular and Medical Pharmacology, Brain Research Institute, \\ David Geffen School of Medicine and Molecular Biology Institute, UCLA, Los Angeles, California, USA.
}

\begin{abstract}
Mutations in the genes PTEN-induced putative kinase 1 (PINK1), PARKIN, and $D J-1$ cause autosomal recessive forms of Parkinson disease (PD), and the Pink1/Parkin pathway regulates mitochondrial integrity and function. An important question is whether the proteins encoded by these genes function to regulate activities of other cellular compartments. A study in mice, reported by Xiong et al. in this issue of the JCI, demonstrates that Pink1, Parkin, and DJ-1 can form a complex in the cytoplasm, with Pink1 and DJ-1 promoting the $\mathrm{E} 3$ ubiquitin ligase activity of Parkin to degrade substrates via the proteasome (see the related article beginning on page 650). This protein complex in the cytosol may or may not be related to the role of these proteins in regulating mitochondrial function or oxidative stress in vivo.
\end{abstract}

Parkinson disease $(\mathrm{PD})$ is the second most common neurodegenerative disorder, and mutations in the genes PTEN-induced putative kinase 1 (PINK1, also known as Parkinson disease 6 [PARK6]), PARKIN (also known as $P A R K 2$ ), and $D J-1$ (also known as PARK7) cause autosomal recessive forms of $\mathrm{PD} /$ parkinsonism. PINK1 encodes a protein with a mitochondrial targeting sequence and a putative serine/threonine kinase domain, and PINK1 is predominantly localized to mitochondria (1). The Parkin protein contains two RING finger motifs, has E3 ubiquitin ligase activity in vitro, and is largely localized to the cytosol (1). The endogenous DJ-1 protein is found in mitochondria and cytosol, but the function of DJ-1 is not entirely clear (1). Studies on the functions of these genes may provide important insights into PD pathogenesis.

\section{Prior studies on protein degradation}

Most PD patients have intraneuronal inclusions in the form of ubiquitin-positive Lewy bodies and Lewy neurites. Given the presence of Parkin in Lewy bodies and the putative role of Parkin as an E3 ligase, much of the initial work on Parkin was focused

Conflict of interest: The authors have declared that no conflict of interest exists.

Nonstandard abbreviations used: PARK2, Parkinson disease 2; PD, Parkinson disease; PINK1, PTEN-induced putative kinase 1; PPD, Parkin/Pink1/DJ-1 (complex); Ub, ubiquitin polypeptide; UPS, ubiquitin-proteasome system.

Citation for this article: J. Clin. Invest. 119:442-444 (2009). doi:10.1172/JCI38619. on its potential role in regulating protein degradation via the ubiquitin-proteasome system (UPS). Ubiquitination is accomplished by covalently linking the ubiquitin polypeptide (Ub) to a lysine residue in a specific protein substrate and requires the sequential action of an E1 activating enzyme, an E2 conjugating enzyme, and an E3 ligase (2). E3 ubiquitin ligases can mediate monoubiquitination (the addition of a single Ub to the substrate protein), multiubiquitination (the addition of multiple single Ubs to different lysine residues in a target protein), and polyubiquitination (in which chains of four or more Ubs are formed by the linkage of $\mathrm{Ub}$ molecules to lysine residues in other Ub molecules) (2). These linkages are most often to lysine 48 (K48) or lysine 63 (K63) of the Ub polypeptide. Proteins to be degraded by the proteasome are largely K48 polyubiquitinated. In contrast, K63 polyubiquitination, as well as monoubiquitination and multiubiquitination, primarily function in non-degradative processes including signal transduction, transcriptional regulation, protein localization, and membrane trafficking (2).

Previous studies suggested that Parkin could function as an E3 ligase for proteasome-mediated protein degradation (3). A handful of substrates of Parkin, including Parkin itself and an $\alpha$-synuclein-interacting protein (Synphilin-1) (4), have been identified in vitro. If Parkin were indeed important in the degradative pathway in vivo, one would expect that the levels of its substrates should increase in Parkin- knockout mice. Unexpectedly, however, most of the substrates studied, including Synphilin-1, did not accumulate in Parkinnull mice (5). In addition, several studies have revealed that Parkin preferentially catalyzes monoubiquitination and K63-linked polyubiquitination of substrates including Synphilin-1 (6-8). These latter observations may offer potential explanations for the lack of substrate accumulation in vivo by implicating Parkin in a non-degradative, proteasome-independent process. Studies in Drosopbila and more recently in mammals have provided important insights into Parkin function, although whether Parkin possesses degradative or non-degradative functions remains to be determined.

\section{Central role of mitochondrial function in PD pathogenesis}

Flies lacking Parkin function show striking defects in mitochondrial morphology that are highly similar, if not identical, to those observed in Pink1 mutants (9-11). Genetic epistasis experiments have demonstrated that Parkin and Pink1 act in a common genetic pathway, with Pink1 positively regulating Parkin $(9,10)$. This Pink1/ Parkin pathway controls mitochondrial integrity at least in part via promotion of mitochondrial fission and/or inhibition of mitochondrial fusion (12-14). Consistent with these findings in Drosopbila, patients with PINK1 or PARKIN mutations have indistinguishable clinical features and also show mitochondrial defects $(1,15)$. Recent studies also suggest that Pink1 and Parkin regulate mitochondrial functions in mammals (16-19). These findings underscore the central importance of the Pink1/Parkin pathway in regulating mitochondrial integrity and function.

Parkin is localized largely in the cytosol, though it can be found within mitochondria or associated with the outer mitochondrial membrane in certain contexts (20-22). Meanwhile, Pink1 has been found within mitochondria in cells and in vivo (9, $23,24)$. The mechanism by which Parkin 